\title{
MadAlegria - Palhaços de hospital: proposta multidisciplinar de humanização em saúde
}

\author{
MadAlegria - Hospital clowns: multidisciplinary \\ approach for health humanization
}

\author{
Key F. Utsunomiya ${ }^{1}$, Elizabeth Alves G. Ferreira², Amanda Manso Oliveira ${ }^{3}$, \\ Henrique Teruo Arai ${ }^{4}$, Maria Aparecida Basile ${ }^{5}$
}

\begin{abstract}
Utsunomiya KF, Ferreira EAG, Oliveira AM, Arai HT, Basile MA. MadAlegria - Palhaços de hospital: proposta multidisciplinar de humanização em saúde / MadAlegria - Hospital clowns: multidisciplinary approach for health humanization. Rev Med (São Paulo). 2012 jul.-set.;91(3):202-8.
\end{abstract}

RESUMO: O objetivo deste artigo é descrever o projeto MadAlegria como uma proposta de humanização em saúde. $O$ MadAlegria é um projeto de extensão universitária da Faculdade de Medicina da Universidade de São Paulo criado em agosto de 2010, com caráter multidisciplinar. Inclui alunos dos cursos de Enfermagem, Fisioterapia, Fonoaudiologia, Medicina, Nutrição e Terapia Ocupacional, os quais desenvolvem atividades culturais, científicas e educacionais relacionadas à humanização do relacionamento com o paciente e com a equipe de saúde. A linguagem utilizada para a comunicação é a lúdica; pela atuação como palhaços de hospital e contadores de história. O projeto tem como finalidade amenizar o ambiente hospitalar e os diferentes cenários de saúde, para os pacientes e para a equipe de trabalho, e influenciar na formação dos alunos da área da saúde com intuito de obter profissionais diferenciados, que possam exercer sua profissão de maneira humanizada e interdisciplinar. Durante os dois anos de existência, foram formados 78 palhaços de hospital, sendo que a formação tem um ano de treinamento, e o curso de contação de histórias foi oferecido para 35 pessoas. A população alvo do projeto é adulto e em dois anos foram realizados 2.020 atendimentos como palhaços no hospital. Os relatórios elaborados pelos alunos após os atendimentos demonstram que há uma mudança positiva na qualidade do contato entre o aluno e o paciente e que o aluno tende a dedicar ao paciente um olhar mais compreensivo e afetivo. Os pacientes referem satisfação e raramente rejeitam a presença do palhaço. É uma oportunidade do aluno da área de saúde se aproximar do paciente sem a obrigação de desempenhar o papel de profissional, mas com a liberdade de ouvir, se sensibilizar com a dor do outro e atuar naquele momento para minimizar a tristeza ou dor ou simplesmente compartilhar aquela experiência de internação hospitalar. O Programa MadAlegria colabora para a discussão da importância de estratégias de humanização no atendimento hospitalar e na formação do profissional de saúde.

DESCRITORES: Humanização da assistência; Assistência ao paciente/psicologia; Trabalhadores voluntários de hospital/psicologia; Narração; Pessoal de saúde/psicologia; Hospitalização; Terapia pela arte.

\footnotetext{
${ }^{1}$ Graduanda do Curso de Fisioterapia - Departamento de Fisioterapia, Fonoaudiologia e Terapia Ocupacional da Faculdade de Medicina da Universidade de São Paulo.

${ }^{2}$ Professora Doutora do Curso de Fisioterapia - Departamento de Fisioterapia, Fonoaudiologia e Terapia Ocupacional da Faculdade de Medicina da Universidade de São Paulo.

${ }^{3}$ Graduanda do Curso de Terapia Ocupacional - Departamento de Fisioterapia, Fonoaudiologia e Terapia Ocupacional da Faculdade de Medicina da Universidade de São Paulo.

${ }^{4}$ Graduando do Curso de Medicina - Faculdade de Medicina da Universidade de São Paulo.

${ }^{5}$ Professora Doutora do Curso de Medicina - Faculdade de Medicina da Universidade de São Paulo.
} 
ABSTRACT: The objective of this article is to describe the project MadAlegria as a multi-disciplinary proposal of humanization in healthcare. MadAlegria is a multidisciplinary extension project of the School of Medicine of University of São Paulo initiated in August 2010. The project includes students of Nursing, Physical Therapy, Speech Therapy, Medicine, Nutrition and Occupational Therapy, who develop cultural, scientific and educational activities related with the humanization of the relationship with the patient and with the health professionals. The students act as hospital clowns and storytellers as a vehicle for the engagement. The project aims to tendering the hospital environment for patients and for the health professionals and to influencing the education of heath care students, seeking to shape differentiated professionals that can work in an interdisciplinary and humanized way. In two years of existence 78 people were trained as hospital clowns and 35 underwent a storytelling

\section{INTRODUÇÃO}

A figura do palhaço é antiga e normalmente associada à diversão das crianças. No ambiente hospitalar a abordagem do palhaço tem como meta auxiliar a criança a se situar e sentir-se incluída naquele ambiente que tem sons, regras e hierarquia específicas ${ }^{1}$. Para os adultos a realidade hospitalar não é menos assustadora, porque significa o rompimento com a rotina de vida e a submissão a um novo conjunto de regras e autoridade, numa situação distante da família e amigos. A permanência no hospital numa situação de internação, mesmo que por poucos dias, é uma experiência estressante.

O estresse psicológico leva à ativação dos eixos hipotálamo - pituitária - adrenal e simpático adrenal. A produção de glicocorticóide e catecolaminas pode influenciar diretamente vários componentes do processo de cicatrização, sendo que há evidências em animais e humanos que as respostas ao estresse psicológico podem retardar a fase de inflamação inicial do processo de cicatrização e altos níveis de ansiedade e depressão podem retardar até quatro vezes mais o tempo de cicatrização $0^{2,3,4}$.

A maioria dos grupos de palhaços de hospital atendem crianças, o que talvez explique o fato da maioria dos estudos científicos serem realizados com elas ${ }^{5,6,7,8}$.

Path Adams ${ }^{9}$ destaca a importância da comunidade cuidar das pessoas, do quanto é importante ter a sensação de pertencimento a um grupo e que o sistema de saúde deve oferecer o correto cuidado e não prometer a cura. Salienta ainda que a relação do profissional de saúde com o paciente é um relacionamento de mão dupla, cque course. In this period the students made 2020 visits as hospital clowns, targeting a population of adult patients. The post visit reports indicate a positive change in the quality of the student-patient contact, as well as a more affective and understanding approach by the student. Patients refer satisfaction and rarely reject the clown's presence. These contacts offer the students the opportunity to get closer to patients with no professional expectations, but with freedom to listen and to empathize with the other's pain and sorrow and eventually help minimizing it. The MadAlegria program supports the discussion on the importance of humanization strategies during hospital care activities as well as in the education of health professionals.

KEYWORDS: Humanization of assistance; Patient care/ psychology; Hospital volunteers/psychology; Narration; Health personnel/psychology; Hospitalization; Art therapy.

modifica, transforma e influencia tanto o paciente quanto o profissional de saúde.

Registros apontam que no Brasil muitos palhaços visitavam hospitais, mas de maneira informal. Em 1991 a abordagem de pacientes por palhaços de hospital começou a ser divulgada no Brasil pela atual ONG Doutores da Alegria. Em 2001 já existia, só no Brasil, mais de 180 grupos de palhaços com atuação em hospitais ${ }^{10}$. Em várias instituições de ensino superior os estudantes têm organizado grupos de palhaços de hospital e apesar dos objetivos serem comuns aos grupos, a metodologia e treinamento são bem diversos. Identifica-se, atualmente, a tendência de desenvolver pesquisas nos projetos e promover intercâmbio de experiências e metodologias. Neste contexto, surge o MadAlegria, que é um projeto de palhaços de hospital e contadores de história vinculado à Faculdade de Medicina da Universidade de São Paulo.

\section{OBJETIVO}

Descrever o Projeto MadAlegria como uma proposta multidisciplinar de humanização em Saúde.

Projeto Mad Alegria

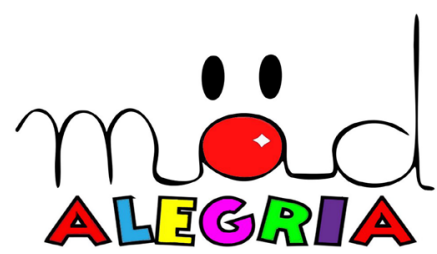

"Seja a mudança que você quer ver no mundo" Mahatma Gandhi 


\section{Histórico}

O MadAlegria é um projeto multidisciplinar de extensão universitária da Faculdade de Medicina da Universidade de São Paulo (FMUSP) e foi criado em agosto de 2010. Conta com o apoio institucional da FMUSP e inclui alunos e docentes dos cursos de Enfermagem, Fisioterapia, Fonoaudiologia, Medicina, Nutrição e Terapia Ocupacional, os quais desenvolvem atividades culturais, científicas e educacionais com enfoque na humanização do relacionamento com paciente e com a equipe de saúde.

\section{Metas do MadAlegria}

O projeto tem como objetivo amenizar o ambiente hospitalar e outros cenários de prática de saúde, promover o trabalho em equipe multidisciplinar e acima de tudo sensibilizar os estudantes para a importância da humanização na relação entre o profissional da saúde e o paciente, incentivando o exercício de cidadania, que é essencial para o equilíbrio social.

O projeto visa desenvolver ações culturais, cientificas e educacionais com foco no conceito de humanização incentivando atividades que envolvem o trabalho em equipe entre estudantes de vários cursos da área da saúde.

A intenção é dar ao estudante a oportunidade de ampliar a sua visão em relação ao paciente, sensibilizando-o sobre a importância da humanização na área da saúde e a responsabilidade que se tem frente ao sofrimento do outro, a fim de formar melhores profissionais de saúde e que tenham um melhor engajamento e responsabilidade no exercício profissional.

\section{Organização}

O Projeto é constituído de uma coordenação discente e uma coordenação docente, ambas de caráter multiprofissional, sendo que a discente se renova a cada ano e tem autonomia para gerir o projeto.

No início do ano é organizado o Curso Introdutório, com duração de três dias, e que tem como público alvo qualquer interessado em conhecer a proposta do MadAlegria. A seguir, há um processo seletivo somente para os alunos dos seis cursos da área da saúde que fazem parte do projeto, e são disponibilizadas 40 vagas para a formação em Palhaços de Hospital, sendo seis para discentes de cada um dos cursos, quatro para profissionais e 20 vagas para a formação de Contadores de Histórias.

\section{Curso de Formação para Palhaço de Hospital}

O curso de formação em palhaço não tem como meta formar atores e sim desenvolver nos estudantes habilidades de comunicação, que facilitem a abordagem lúdica do paciente. $O$ treinamento oferecido em 2012 teve apoio institucional da FMUSP e foi ministrado pela Organização Não Governamental (ONG) Doutores da Alegria. Em sala de aula sempre há dois professores e um observador para registro das aulas, ocasião em que são vivenciadas técnicas para treino da atenção, desinibição, cumplicidade entre os colegas e abordagem dos pacientes. A música, o improviso, a capacidade de observar o outro, e rapidamente se conectar ao seu espaço e momento, são estratégias amplamente discutidas e vivenciadas no treinamento. Os aspectos teóricos sobre palhaços de hospital e o perfil deste tipo de personagem também são discutidos. No decorrer do curso, os estudantes são incentivados a identificar peças de vestuário que possam caracterizar o seu palhaço, o qual recebe nome e muitas vezes sobrenome. Em todas as aulas há um período reservado para a discussão das sensações e impressões dos participantes, o que favorece a sensação de pertencimento e vínculo com o grupo. Fora das aulas, a comunicação do grupo é feita pessoalmente ou por meios eletrônicos, mas sempre incentivando o contato entre seus membros. A formação tem duração de um ano, com aulas semanais de três horas de duração e estágio supervisionado no Hospital das Clínicas da FMUSP e no Instituto do Câncer do Estado de São Paulo (ICESP). No primeiro semestre ocorrem as aulas e no segundo semestre são intercaladas aulas e atendimentos supervisionados no hospital. Os alunos são subdivididos em grupos menores e, sob a supervisão de um palhaço formado pelo projeto no ano anterior, são encaminhados para atendimentos que duram em media três horas, sendo que cada grupo fica responsável por atender uma determinada ala hospitalar ou instituição de saúde. Nesta fase, as aulas tem o intuito de rever estratégias de palhaços, mas também promover a troca de experiências entre os estudantes e dar suporte a sentimentos de conflito ou insegurança percebidos pelo aluno durante o atendimento.

As Figuras 1 e 2 apresentam as turmas I e II dos cursos de formação de palhaço de hospital. 


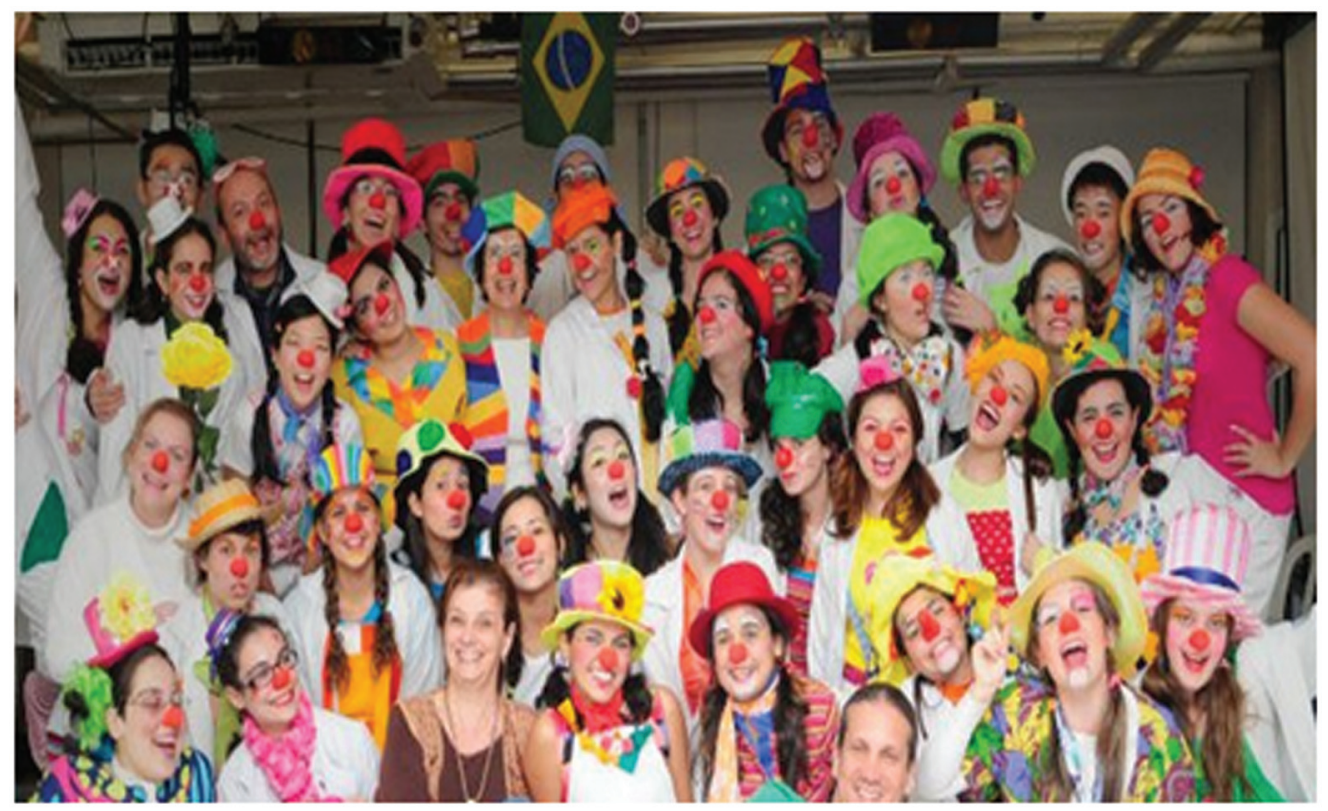

Figura 1. Curso de formação para palhaços de hospital 2011

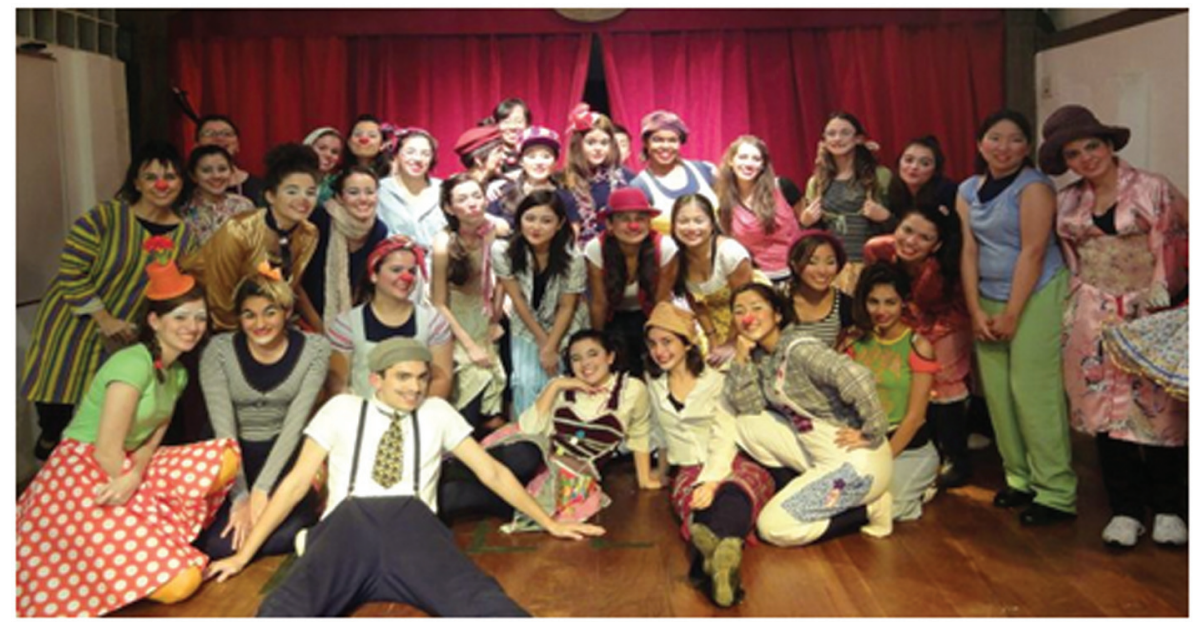

Figura 2. Curso de formação para palhaços de hospital 2012

\section{Curso de Formação para Contador de Histórias}

O treinamento para Contador de Histórias ocorre com encontros mensais de seis horas de duração aos sábados, durante um ano e é ministrado por uma Contadora de Histórias com ampla experiência em atuação hospitalar. O curso, com aulas teóricas e práticas, tem como objetivo discutir aspectos da oralidade, bem como, desenvolver habilidades para narração de histórias e compreender a função de recursos como bonecos, instrumentos e música na narrativa. Contar histórias é um recurso que facilita a aproximação com o paciente e pode ser usado nas diversas faixas etárias, sendo que a escolha correta da história ou da música pode facilitar muito a empatia com o paciente no ambiente hospitalar e nos diferentes cenários.

\section{Os Atendimentos}

São realizados no hospital uma vez por semana. Os integrantes do projeto podem e são incentivados a caracterizar seu personagem, mas no hospital sempre é obrigatória a utilização do jaleco com o logotipo do projeto.

As visitas ao hospital tem em média duração de três horas e são realizadas em duplas de palhaços. Inicialmente, verifica-se com o Serviço de Enfermagem quais pacientes podem ser visitados e se há 
algum paciente que deve ser poupado em função de seu estado clínico ou emocional. Pacientes em isolamento também são atendidos pelos palhaços, desde que seja permitido/orientado pelo Serviço de Enfermagem. Até o presente momento o foco do projeto é o atendimento de adultos, incluindo idosos, grávidas de alto risco e pacientes com disfunções neurológicas, moléstias infecciosas, doenças dermatológicas ou câncer.

Ao chegar ao quarto, o palhaço pede consentimento ao paciente para entrar e é reservado ao paciente o direito de aceitar ou não a intervenção.

A abordagem lúdica do palhaço não se limita ao paciente internado, mas se estende ao acompanhante e à equipe de profissionais do serviço.

Após os atendimentos os alunos fazem um relatório salientando os fatos mais relevantes, bem como sensações e impressões ocorridas.

Além disso, o grupo é convidado a participar de eventos específicos como; Oficinas em congressos e comemorações diversas, que estejam alinhadas com os objetivos do projeto. Nestas situações a figura do palhaço é importante para facilitar a integração das pessoas e sensibilizar sobre a importância da humanização no cenário de ensino e prática de saúde.

\section{RESULTADOS}

Em 2011 terminaram a formação de Palhaço 35 estudantes, uma docente e dois profissionais de saúde. Em 2012 serão formados 40 novos palhaços, sendo 37 estudantes e três docentes. Também serão formados 12 contadores de história.

Em 2011 foram atendidos 1020 pacientes em 43 visitas realizadas ao hospital, e somente no primeiro semestre de 2012 foram realizadas 50 visitas, totalizando 999 pacientes atendidos.

Os dados coletados no primeiro semestre de 2012 permitem observar o gênero e a distribuição em faixas etárias dos pacientes atendidos nos leitos (Figura 3) e seus acompanhantes (Figura 4).

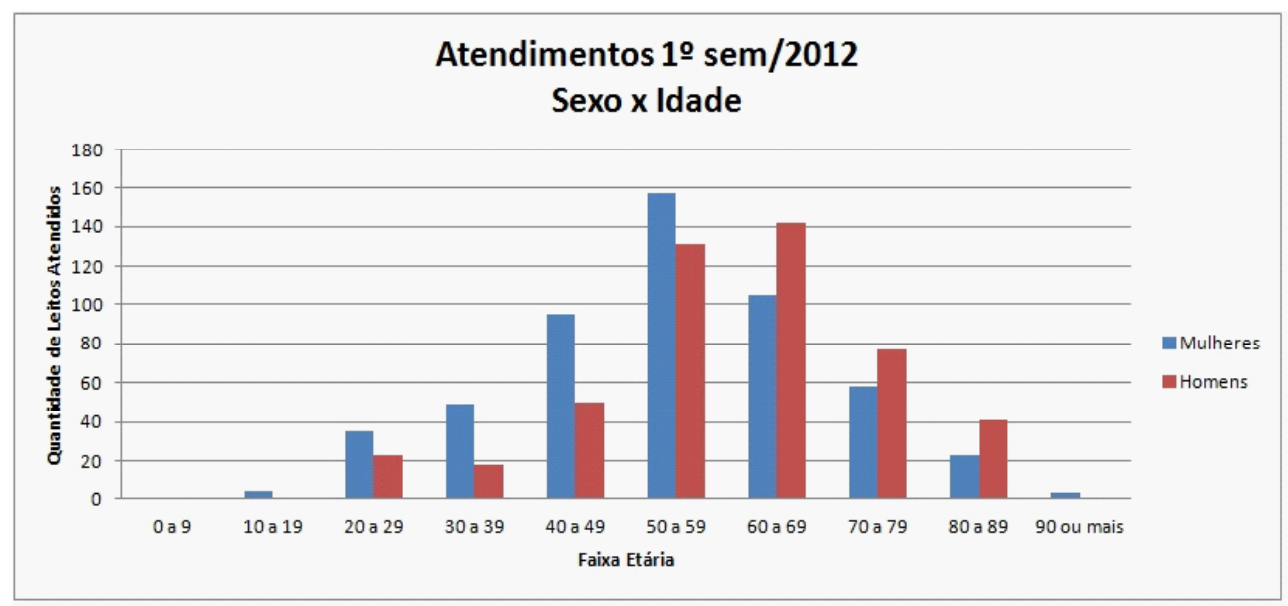

Figura 3. Gênero e distribuição por faixa etária dos pacientes atendidos pelos palhaços nos leitos no primeiro semestre de 2012

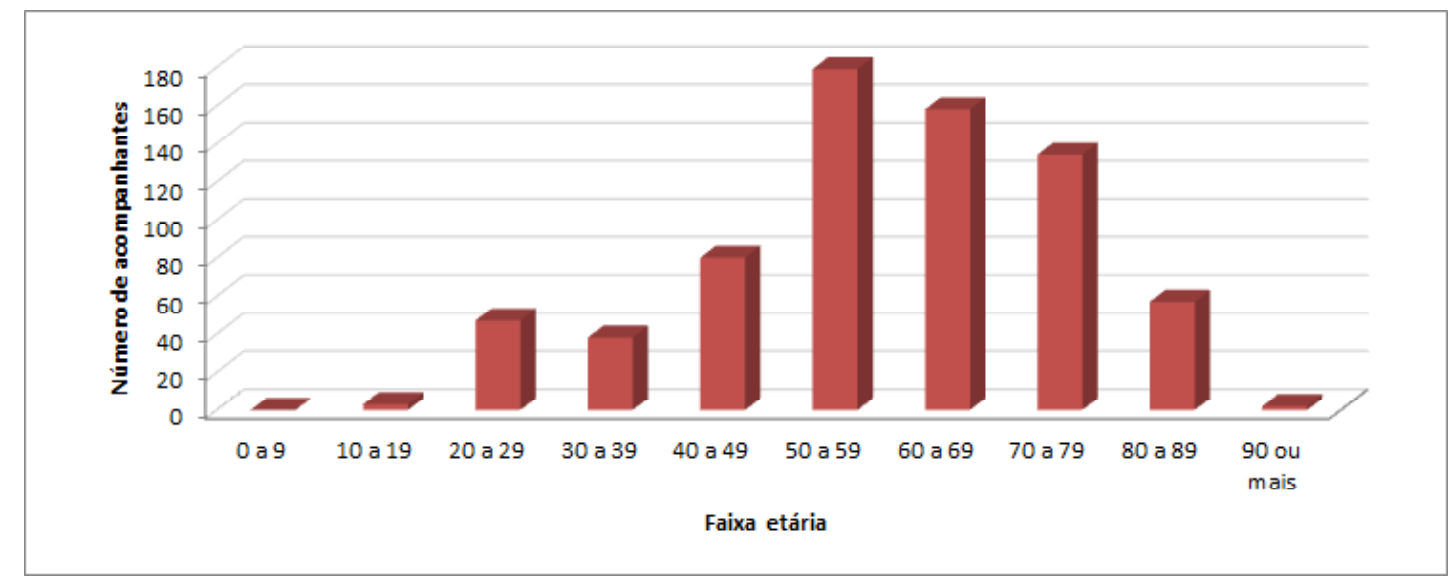

Figura 4. Distribuição por faixa etária dos acompanhantes dos pacientes atendidos pelos palhaços no primeiro semestre de 2012 
Os relatórios apresentados pelos estudantes descrevem situações e discutem temas relevantes para a formação do profissional de saúde, como por exemplo; a dificuldade em lidar com o paciente terminal e a morte, casos em que estabelecer o vínculo com o paciente é muito fácil e outras situações no mesmo dia em que a mesma tarefa é muito difícil, a relação com os familiares, com a equipe profissional, a necessidade de ser ágil no pensar e tomar decisões, enfim, participar como membro atuante do cenário hospitalar.

\section{DISCUSSÃO}

A implantação de um programa como o MadAlegria é trabalhosa, mas a sua continuidade depende, principalmente, do compromisso de seus participantes, e do estabelecimento de metodologias de avaliação crítica e de ajustes, que permitam fidelidade aos objetivos da proposta. Em dois anos de existência o MadAlegria passou por uma reestruturação importante, sobretudo no formato do treinamento. $\mathrm{O}$ apoio institucional foi determinante para que algumas mudanças fossem implementadas.

A formação de novos palhaços e contadores de história é essencial para a continuidade do projeto e o aumento do número de docentes envolvidos é um fator promissor. Os estudantes tem um período de permanência na Universidade menor do que os professores, e esta mescla de docentes e discentes pode colaborar para que a história do projeto e suas metas sejam conservadas.

O treinamento de um ano pode parecer longo, mas é importante para estimular a disciplina entre os interessados em realizar o trabalho voluntário, manter o compromisso com uma proposta em que os resultados não são imediatos, é necessário se preparar para realizar a tarefa com devido engajamento. Atualmente os jovens tendem a ser mais imediatistas em um mundo cada vez mais virtual e eletrônico. Assumir compromissos que implicam em preparo longo valoriza a responsabilidade, tão importante para 0 desenvolvimento de um bom profissional.

Em relação aos pacientes atendidos no primeiro semestre de 2012, a quase totalidade é constituída por adultos, com predomínio nas faixas de idade entre $40 \mathrm{e}$ 79 anos. A internação é uma experiência que, normalmente, fragiliza o paciente e os idosos tendem a ser mais carentes, vivem de suas reminiscências e gostam de dividir com os outros os fatos que marcaram suas vidas. Sentem-se valorizados quando são ouvidos com atenção, o que melhora sua auto-estima e pode repercutir em melhora acentuada no seu processo de reabilitação ${ }^{11}$.

Muitos grupos de palhaços de hospital focam no atendimento infantil, o que gera mais pesquisas com crianças, mas resultados destes estudos podem ser utilizados para discutir a função do humor e da alegria no hospital. Koler ${ }^{12}$ descreve um estudo em que crianças entre 4 a 12 anos responderam entrevistas semiestruturadas sobre suas experiências com palhaços no hospital. Os resultados apontaram para temas como o humor, e que com os palhaços elas tinham a permissãopara rirem e serem felizes. O humor atua como uma estratégia para lidar com o estresse e os sintomas psicológicos relacionados a situações negativas ${ }^{12}$. $\mathrm{O}$ trabalho dos palhaços pode contribuir para que os pacientes desviem, mesmo que de forma temporária, o foco de sua dor e de sua doença e se permitam a diversão, ainda que no ambiente hospitalar.

O humor ajuda a manter as relações entre as pessoas, facilita a criação e a manutenção de víncu$\operatorname{los}^{13}$, tão essenciais para o trabalho em equipe e entre o profissional de saúde e o paciente, além de diminuir as barreiras entre as pessoas e tornar a comunicação mais ágil e efetiva.

No riso e na alegria o palhaço, o paciente, os acompanhantes e os profissionais de saúde se encontram; e, este momento, é uma oportunidade potencializadora de trocas, de atitude de vida, porque o riso é uma conduta ativa, uma ação voluntária ${ }^{10,14}$.

Uma característica muito específica do MadAlegria é ser multiprofissional. Pelas Diretrizes Curriculares Nacionais espera-se do profissional de saúde que ele tenha habilidade de trabalhar em equipe, que ele seja criativo, criador, agente de mudança, mas na prática o estudante é pouco exposto a esta realidade na graduação, uma vez que o objetivo central desta fase é instrumentar tecnicamente o estudante para que ele seja um profissional competente na sua área de formação. O MadAlegria possibilita aos participantes ter uma experiência intensa de trabalho multiprofissional ainda na graduação, conhecendo melhor as outras profissões e suas interfaces, ao mesmo tempo que vivencia a equipe multiprofissional em atendimento hospitalar. A formação de um profissional de saúde responsável por suas atitudes, empático, capaz de trabalhar em equipe e consciente da prática dos conceitos de humanização está de acordo com os preceitos das Diretrizes Curriculares Nacionais e do Humaniza SUS.

Apesar dos pacientes poderem rejeitar a intervenção do palhaço no hospital, isto raramente ocorre, a figura lúdica e descompromissada do palhaço normalmente é aceita e transforma rapidamente o ambiente do quarto.

\section{CONCLUSÃO}

O projeto ainda é jovem, mas os resultados 
obtidos até o momento demonstram que tanto o palhaço de hospital como o contador de histórias são recursos que podem ser utilizados em beneficio do paciente e em beneficio dos alunos da área de saúde. A alegria pode ser uma forma de abordagem e de humanização das relações.

Agradecimentos: À Diretoria da Faculdade de Medicina da Universidade de São Paulo, à Comissão de Cultura e extensão FMUSP, à Fundação Faculdade de Medicina, ao Hospital das Clínicas da FMUSP, e ao Instituto do Câncer do Estado de São Paulo (ICESP) pelo apoio ao projeto.

\section{REFERÊNCIAS}

1. Oppenheim D, Simonds C, Hartmann O. Clowning on children's wards. Lancet. 1997;350:1838-40.

2. Powell ND, Allen RG, Hufnagle AR, Sheridan JF, Bailey MT. Stressor-induced alterations of adaptive immunity to vaccination and viral pathogens. Immunol Allergy Clin N Am. 2011;(31):69-79.

3. Gouin, JP, Kiecolt-GlaserJK. The impact of psychological stress on wound healing: methods and mechanisms. Immunol Allergy Clin North Am. 2011;31(1):81-93.

4. Cole-King A, Harding KG. Psychological factors and delayed healing in chronic wounds. Psychosom Med. 2001;63:216-20.

5. Bertini M, Isola E, Paolone G, Curcio G. Clowns benefit children hospitalized for respiratory pathologies. Evid Based Complement Alternat Med. 2011:879125. doi: 10.1093/ecam/neq064.

6. Vagnoli L, Caprilli S, Messeri A. Parental presence, clowns or sedative premedication to treat preoperative anxiety in children: what could be the most promising option? Paediatr Anaesth. 2010;20(10):937-43.

7. Fernandes SC, Arriaga P. The effects of clown intervention on worries and emotional responses in children undergoing surgery. J Health Psychol. 2010;15(3):405-15.

8. Kingsnorth S, Blain S, McKeever P. Physiological and emotional responses of disabled children to therapeutic clowns: a pilot study. Evid Based Complement Alternat Med. 2011:732394.

9. Adams P. Medicine as a Vehicle for Social Change. J Alternat Complement Med. 2005;11(4):578-82.

10. Masetti, M. Boas Misturas: a ética da alegria no contexto hospitalar. São Paulo: Palas Athena; 2003.

11. Sachetto, RM. A comunicação como estratégia complementar no cuidado fisioterapêuticogeriátrico [citado 5 nov. 2012]. Disponível em: http:// encipecom.metodista.br/mediawiki/index. php/A_comunica\%C3\%A7\%C3\%A3o_como_ estrat\%C3\%A9gia_complementar_no_cuidado_ fisioterap\%C3\%AAutico_geri\%C3\%A1trico. 05/11/2012.

12. Koller D, Gryski C. The life threatened child and the life enhancing clown: towards a model of therapeutic clowing. Evid Based Complement Alternat Med. 2008;5(1):17-25.

13. Dean RAK. Expressing Sensibilities: healing functions of humour in palliative care. In: Litvack AD. Making sense of: stress, humour and healing. Oxford: Inter-Disciplinary Press; 2006.

14. Masetti M. Soluções de palhaços. 7a ed. São Paulo: Palas Athena; 2008. 